\title{
NECESSARY AND SUFFICIENT CONDITIONS FOR A STATISTICAL PROBLEM TO BE INVARIANT UNDER A LIE GROUP1
}

\author{
By David R. Brillinger
}

\section{Bell Telephone Laboratories and Princeton University}

1. Introduction and summary. Although a great deal has been written concerning the theory of tests, decisions and inference for statistical problems invariant under the action of some group, (see for example [4]-[7], [9], [12]-[14], [16]), no great amount of literature exists concerning the problem of discerning whether or not a given problem is actually invariant under some group. In fact the literature seems to consist of one abstract [8] and one paper [15].

In this paper necessary and sufficient conditions are developed that a statistical problem must satisfy in order that it be invariant, in a precise sense to be defined later, under a fairly general class of transformation groups, Lie transformation groups. It must be added, however, that the sufficient conditions are to some extent tautological. In addition two methods of actually constructing the group, if it can be shown to exist, are given, and the main theorem is illustrated by a variety of examples.

One of the examples yields the interesting result that the fiducial distribution of the correlation coefficient derived from a sample from a bivariate normal distribution by R. A. Fisher is not a Bayes' distribution for any prior distribution.

2. The definition of a Lie group. The following definitions are essential to what follows.

A group $G$ is said to be a transformation group on the set $E$ if $G$ is a subgroup of the group of all $1-1$ mappings of $E$ onto itself.

Let $F$ be any subset of $E$, then the set of all elements $g x$ for $g \varepsilon G, x \varepsilon F$, is called the orbit of $F$ under $G$.

A topological group is a group which is also a Hausdorff space and the maps,

(i) $g, h \rightarrow g h: G \times G \rightarrow G$

(ii) $g \rightarrow g^{-1}: G \rightarrow G$

are continuous.

Let $G$ be a topological group and $X$ a Hausdorff space. Assume that for each $g \varepsilon G$ there exists a homeomorphism of $X$ onto $X, T_{g}: X \rightarrow X: x \rightarrow \varphi(x, g)=$ $T_{g} x$ such that

(i) $T_{e}=$ identity $=I, e$ the identity of $G$

(ii) $T_{g_{1}} T_{\theta_{2}}=T_{g_{1} \theta_{2}}$

(iii) The function $(g, x) \rightarrow \varphi(x, g): G \times X \rightarrow X$ is continuous, then $\varphi: G \times X$

Received November 3, 1961; revised December 27, 1962.

1 Part of this research was carried out while the author was at the London School of Economics with the support of a Research Training Fellowship of the Social Science Research Council. 
$\rightarrow X$ is called a topological transformation group of $G$ acting on $X$ by the function $\varphi$.

If in addition,

(iv) $T_{g}=I \Leftrightarrow g=e$, then $G$ acts effectively on $X$.

A topological transformation group $G$ is transitive on a space $X$ if for every $x, y \varepsilon X$ there is a $g \varepsilon G$ such that $g x=y$. The space $X$ is then called a homogeneous space.

In what follows, differentiable should always be understood to refer to that of class $C^{\infty}$.

An $n$-dimensional manifold $M^{n}$, is a Hausdorff space which is locally $n$-dimensional Euclidian at each point. Because $M^{n}$ is locally Euclidian, every point $p$ has a neighborhood with a system of coordinates $x_{1}^{p}, \cdots, x_{n}^{p}$.

A manifold, together with a set of overlapping coordinate systems, which cover the entire manifold and has the property that the transformation between any two overlapping coordinate systems is differentiable is called a differentiable manifold.

A Lie group is a topological group which is also a differentiable manifold and such that,

(i) $g, h \rightarrow g h: G \times G \rightarrow G$

(ii) $g \rightarrow g^{-1}: G \rightarrow G$ are differentiable maps.

Let $G$ be a Lie group and $M^{n}$ a differentiable manifold. Assume that for each $g \varepsilon G$ there exists a diffeomorphism of $M^{n}$ onto $M^{n}, T_{g}: M^{n} \rightarrow M^{n}: x \rightarrow \varphi(x, g)$ such that:

(i) $\varphi: G \times M^{n} \rightarrow M^{n}$ is a topological transformation group,

(ii) The function $(g, x) \rightarrow \varphi(x, g): G \times M^{n} \rightarrow M^{n}$ is differentiable, then $\varphi: G \times M^{n} \rightarrow M^{n}$ is a Lie transformation group.

Examples of Lie transformation groups include all affine transformations of $R^{n}$ and all orthogonal transformations of $R^{n}$.

Associated with any Lie transformation group $G: M \rightarrow M$, is a set of infinitesimal generators defined as follows: the mapping $(g, x) \rightarrow g x=y, g \varepsilon G, x, y \varepsilon M$ is differentiable. Let $(g)=\left(g^{1}, g^{2}, \cdots, g^{r}\right)$ denote a set of coordinates at $e \varepsilon G$ and $(x)=\left(x^{1}, x^{2}, \cdots, x^{n}\right)$ be a set of coordinates at some point $x_{0}$ of $M$. The mapping can now be expressed as follows, $y^{\alpha}=\varphi^{\alpha}(g, x) \alpha=1, \cdots, n$, with $\varphi^{\alpha}$ differentiable. Put

$$
\psi_{i}^{\alpha}(x)=\left[\partial \varphi^{\alpha}(g, x) / \partial g^{i}\right]_{g=e}
$$

$X_{i}=\sum_{\alpha} \psi_{i}^{\alpha}(x)\left(\partial / \partial x^{\alpha}\right)$ is called an infinitesimal generator of the Lie transformation group $G$ over the manifold $M$. It is a differential operator acting on the space of differentiable functions of $x$.

Lie groups appear to be natural groups for a statistician to be concerned with. They are locally compact and hence possess the Haar measure required in the Hunt-Stein Theorem [9] or in Fraser's work on fiducial probability, [4], [5] for example. The transformations they induce are defined on a manifold, the type of 
space, the sample space or parameter space usually is in a statistical problem. Finally the transformations induced by a Lie group are continuous, a property that seems sensible for transformations applied to random variables or parameters in a real problem.

3. An invariant statistical problem. In what follows the essential components of a statistical decision problem will be defined and necessary and sufficient conditions that such a problem be invariant given, for most statistical problems are generally made up of some of these basic components.

Let $X$ be the set of all experimental outcomes, $B_{\mathfrak{X}}$ a $\sigma$-algebra of subsets of $x, \Theta$ the set of states of nature, and $P(\cdot, \cdot)$, the specification, a real-valued function defined on $B_{\mathfrak{X}} \times \Theta$, such that for each $\theta \varepsilon \Theta, P(\cdot, \theta)$ is a probability measure on $B_{\mathfrak{x}}$. Let $A$ be the set of actions available to the statistician, $B_{A}$ a $\sigma$-algebra of subsets of $A$, and $L$, the loss function, a real-valued function on $\Theta \times A \times X$ such that $L(\theta, a, x)$ is the loss to the statistician when he takes action $a$, after observing $x$, and $\theta$ is the true state of nature. $L$ is assumed jointly measurable in $a$ and $x$. Let $D$ be the class of randomized decision functions from $x \times B_{A}$ into the unit interval, such that for each $x, \delta_{x}$ is a probability measure on $\left(A, B_{A}\right)$ and such that for fixed $a, \delta_{a}$ is a measurable function of $x$. The associated risk $R(\theta, \delta)$ for $\theta \varepsilon \Theta$ is defined by,

$$
R(\theta, \delta)=\iint L(\theta, a, x) d(x, a) d P(x, \theta) .
$$

The above statistical decision problem is said to be invariant under the group $G$ if,

(i) $G$ is a transformation group on each of $x, \Theta, A$,

(ii) $g: x \rightarrow x$ and $g: A \rightarrow A$ are each measurable transformations on the respective spaces,

(iii) $P(g B, g \theta)=P(B, \theta), B \varepsilon B_{\mathfrak{x}}, \theta \varepsilon \Theta$ where $g$ belongs to the transformation group $G$ on $\Theta \times A \times x$ obtained by defining $g(\theta, a, x)=(g \theta, g a, g x)$ and

(iv) $L(g \theta, g a, g x)=L(\theta, a, x)$.

If in addition $g \delta=\delta$ for all $g \varepsilon G$ the decision procedure $\delta$ will be said to be invariant under $G$.

The goal of this paper is to find necessary and sufficient conditions that a statistical decision problem, in which $\Theta, A$, and $X$ are differentiable manifolds, must satisfy, in order that it be invariant under the action of some Lie transformation group.

The following lemmas will be required later.

LEMMA 1. If $G$ is a Lie transformation group over the manifolds, $\Theta, A, x$ its infinitesimal generators, when it is considered a Lie transformation group over $\Theta \times A \times x$, are of the form,

$$
X_{i}=\sum_{\alpha} \psi_{i}^{\alpha}(\theta) \frac{\partial}{\partial \theta^{\alpha i}}+\sum_{\beta} \rho_{i}^{\beta}(a) \frac{\partial}{\partial a^{\beta}}+\sum_{\gamma} \sigma_{i}^{\gamma}(x) \frac{\partial}{\partial x^{\gamma}},
$$

i.e., $X_{i}$ is the sum of the infinitesimal generators of $G$ over $\Theta, A, \mathfrak{X}$ respectively. 
Proof. This is an immediate result of the particular nature of,

$$
G: \Theta \times A \times x \rightarrow \Theta \times A \times x .
$$

Lemma 2. Let $F$ be any real-valued differentiable function defined on $\Theta \times A \times x$. If $F$ is invariant under the Lie group $G$, i.e., $F(g \theta, g a, g x)=F(\theta, a, x)$ for all $g \varepsilon G$, then $X_{i} F \equiv 0$, where the $X_{i}$ are the infinitesimal generators of $G$. If $X_{i} F \equiv$ 0 and $G$ is connected, then the converse is true, namely $F$ is invariant under $G$.

Proof. If $F(\theta, a, x)$ is invariant under $G$ then $F(g \theta, g a, g x)$ is independent of $g$. That is, $\partial F(g \theta, g a, g x) / \partial g^{i}=0$. But $\left[\partial F(g \theta, g a, g x) / \partial g^{i}\right]_{g=e}=X_{i} F$, thus $X_{i} F \equiv 0$ for all $i$.

Conversely suppose $X_{i} F \equiv 0$ for all $i$. Now,

$$
F\left(g h \theta_{0}, g h a_{0}, g h x_{0}\right)=F\left(g \theta_{1}, g a_{1}, g x_{1}\right),
$$

where $\theta_{1}=h \theta_{0}, a_{1}=h a_{0}, x_{1}=h x_{n}$. Thus

$$
\left[\partial F\left(g h \theta_{0}, g h a_{0}, g h x_{0}\right) / \partial g^{j}\right]_{g=e}=\left[X_{j} F^{n}\right]_{j=\hat{\vartheta}_{1}, \dot{\omega}=\bar{a}_{1}, \dot{x}=\bar{x}_{1}}=0 .
$$

Now

$$
\begin{aligned}
\partial F\left(h \theta_{0}, h a_{0}, h x_{0}\right) / \partial h^{j}=\left[\partial F\left(g h \theta_{0}, g h a_{0}, g h x_{0}\right) / \partial(g h)^{j}\right]_{g=e} \\
=\sum_{i}\left\{\left[\partial F\left(g h \theta_{0}, g h a_{\hat{v}}, g h x_{0}\right) / \partial g^{i}\right] \cdot\left[\partial g^{i} / \partial(g h)^{j}\right]\right\}_{g=e} .
\end{aligned}
$$

Thus $\partial F\left(h \theta_{0}, h a_{0}, h x_{0}\right) / \partial h^{j}=0$ for all $j$, and since $G$ is connected $F$ is invariant.

The main theorem of the paper is the following.

Theorem. Let the random variable $X$ have a density $f(x, \theta)$ with respect to Lebesgue measure, and let $f$ and the loss function $L$ be differentiable functions. If the statistical decision problem is invariant under a Lie transformation group $G$ then,

(1) $\sum_{\alpha} \psi_{i}^{\alpha}(\theta) \frac{\partial L}{\partial \theta^{\alpha}}+\sum_{\beta} \rho_{i}^{\beta}(a) \frac{\partial L}{\partial a^{\beta}}+\sum_{\gamma} \sigma_{i}^{\gamma}(x) \frac{\partial L}{\partial x^{\gamma}}=0 \quad$ for all $i$,

$$
\sum_{\alpha} \psi_{i}^{\alpha}(\theta) \frac{\partial \log f}{\partial \theta^{\alpha}}+\sum_{\gamma} \sigma_{i}^{\gamma}(x) \frac{\partial \log f}{\partial x^{\gamma}}=-\sum_{\gamma} \frac{\partial \sigma_{i}^{\gamma}(x)}{\partial x^{\gamma}} \quad \text { for all } i,
$$

where the linear differential operators

$$
X_{i}=\sum_{\alpha} \psi_{i}^{\alpha}(\theta) \frac{\partial}{\partial \theta^{\alpha}}+\sum_{\beta} \rho_{i}^{\beta}(a) \frac{\partial}{\partial a^{\beta}}+\sum_{\gamma} \sigma_{i}^{\gamma}(x) \frac{\partial}{\partial x^{\gamma}}
$$

$\alpha=1, \cdots, s ; \beta=1, \cdots, t ; \gamma=1, \cdots, u ; i=1, \cdots, v$ are the infinitesimal generators of $G$.

Conversely, if there exist differentiable functions, $\psi_{i}^{\alpha}(\theta), \rho_{i}^{\beta}(a), \sigma_{i}^{\gamma}(x) ; \alpha=1$, $\cdots, s ; \beta=1, \cdots, t ; \gamma=1, \cdots, u ; i=1, \cdots, v$ such that

(1) and (2) above are satisfied

$\left(2^{\prime}\right)$ there exist constants $c_{j k}^{i} i, j, k=1, \cdots, r$ such that $X_{k} X_{j}-X_{j} X_{k}=\sum_{i} c_{j k}^{i} X_{i}$ the $X_{i}$ being given by (3) 
$\left(3^{\prime}\right)$ the $X_{i}$ generate a connected group $G$, then, the statistical problem defined previously is invariant under $G$.

Proof. The measure induced by the density function $f(x, \theta)$ is invariant under the action of $G$, if and only if $f(x, \theta)=f(g x, g \theta)$ Det $\left(\partial \varphi^{\alpha}(g, x) / \partial x^{\gamma}\right)$.

Differentiating this relation with respect to $g^{i}$ and setting $g=e$ one obtains,

$$
0=X_{i} f+f\left[\sum_{\gamma}\left(\partial \sigma_{i}^{\gamma}(x) / \partial x^{\gamma}\right)\right]
$$

This is equivalent to (2). (1) follows immediately from Lemma 2, completing the proof of the necessary part of the theorem.

It was stated in the introduction of the paper that the sufficient conditions that would be developed were somewhat tautological. The following discussion should indicate the reason for this statement.

In the second lemma it was shown that if the differentiable function $F(\theta, a, x)$ is invariant under the Lie group $G$, then it must be such that $X_{i} F=0$ for $X_{i}$ any infinitesimal generator of $G$. It is not however true that if a function $F$ is such that $X_{i} F \equiv 0$ for a set of linear differential operators of the form under consideration, then there is a Lie group of transformations $G$ under which $F$ is invariant. The linear operators may not generate a (global) group of transformations, but only a local one (i.e., one for which inverses etc. are only defined in a neighborhood), or perhaps nothing significant at all. The condition $\left(2^{\prime}\right)$ above is the necessary and sufficient condition that a local group be generated (this is Lie's Second Fundamental Theorem). Necessary and sufficient conditions have been given for a global group to be generated (see [11]), however these conditions do not seem to be easy to apply. If one does have a particular set of linear operators satisfying $\left(2^{\prime}\right)$ the most efficient method of finding out if they generate a global group appears to be to generate the local group and then to check to see if it is actually global. Two methods of doing this follow later.

The sufficiency part of the theorem relative to the loss function now follows from the above considerations and Lemma 2. To complete the proof of the theorem it must now be shown that the probability measure induced by $f(x, \theta)$ is invariant or that, $f(x, \theta)=f(h x, h \theta)$ Det $\left(\partial \varphi^{\alpha}(h, x) / \partial x^{\gamma}\right)$ for all $h$.

Now,

$$
\begin{aligned}
& \partial f(h x, h \theta) / \partial h^{j}=\left[\partial f(g h x, g h \theta) / \partial(g h)^{j}\right]_{g=e} \\
& \quad=\sum_{i}\left\{\left[\partial f(g h x, g h \theta) / \partial g^{i}\right] \cdot\left[\partial g^{i} / \partial(g h)^{j}\right]\right\}_{g=e}=\sum_{i}\left[\partial g^{i} / \partial(g h)^{j}\right]_{g=e}\left[X_{i} f\right]_{x=x_{1}, \theta=\theta_{1}}
\end{aligned}
$$

where $x_{1}=h x, \theta_{1}=h \theta$.

Similarly,

$\left(\partial / \partial h^{j}\right) \operatorname{Det}\left(\partial \varphi^{\alpha}(h, x) / \partial x^{\gamma}\right)$

$$
=\sum_{i}\left[\partial g^{i} / \partial(g h)^{j}\right]_{\theta=e}\left(\sum_{\gamma} \sigma_{i}^{\gamma}(h x)\right) \operatorname{Det}\left(\partial \varphi^{\alpha}(h, x) / \partial x^{\gamma}\right) .
$$


Using the above relations and (2),

$$
\frac{\partial}{\partial h^{j}}\left\{f(h x, h \theta) \operatorname{Det}\left(\partial \varphi^{\alpha}(h, x) / \partial x^{\gamma}\right)\right\}=0 .
$$

Therefore $f(h x, h \theta)$ Det $\left(\partial \varphi^{\alpha}(h, x) / \partial x^{\gamma}\right)$ is a constant with respect to $h$, and setting $h=e$, and using the fact that $G$ is connected, it equals $f(x, \theta)$. The proof of the theorem is now completed.

The theorem has the following corollary for the case in which $X$ is a real random variable with cdf $F(x)$.

Corollany. Let $X$ be a real random variable with c.d.f. $F(x, \theta)$. The statistical problem is invariant under the connected group $G$ if and only if $F_{x} / F_{\theta}=a(\theta) / b(x)$ i.e., $F_{x} / F_{\theta}$ factors into a function of $x$ times a function of $\theta$ and $b(x)(\partial / \partial x)+$ $a(\theta)(\partial / \partial \theta)$ generates $G$.

Proof. This corollary follows immediately from Lemma 2 and the fact that for a real random variable invariance of the probability measure and invariance of the c.d.f. are equivalent. This corollary is inherent in [10].

The reader will have noted that derivatives of all orders have been assumed to exist in the definition of a Lie transformation group. One can in fact proceed with fewer derivatives than this. Minimal conditions are a topic of current research in the theory of Lie groups.

Two methods of actually generating the local group from a given set of linear operators now follow:

Method 1. Let the local group $G$ be generated by the $r$ linear operators $X_{1}$, $\cdots, X_{r}$. If $\bar{x}=g(x)$, then,

$$
\bar{x}^{i}=\exp \left(g^{1} X_{1}+g^{2} X_{2}+\cdots+g^{r} X_{r}\right) x^{i} \text { for } i=1, \cdots, n,
$$

i.e.,

$$
\bar{x}^{i}=\sum_{m}\left[\left(g^{1} X_{1}+\cdots+g^{r} X_{r}\right)^{m} / m !\right] x^{i}
$$

Method 2. Let the local group $G$ be generated by $X_{1}, \cdots, X_{r}$ once again. Find the integrals $\varphi_{1}, \cdots, \varphi_{n}$ of $\left(g^{1} X_{1}+\cdots+g^{r} X_{r}\right) u=1$ then solve

$$
\varphi_{i}\left(\bar{x}^{1}, \cdots, \bar{x}^{n}\right)=\varphi\left(x^{1}, \cdots, x^{n}\right)+1 \quad i=1, \cdots, n
$$

for $\bar{x}$.

The following example illustrates the first method and the corollary.

Example 1. Suppose one is concerned with the Pareto distribution $F(x)=$ $1-\left(1 / x^{\alpha}\right) x \geqq 1 \alpha>0$, i.e., one wishes to find if it is invariant under some group. Now $F_{x} / F_{\alpha}=(\alpha / x) \ln x$, i.e., $F_{x} / F_{\alpha}$ factors as required; therefore $F(x)$ may be invariant under some local Lie transformation group. Let us try to generate this group by Method 1 given above.

Its infinitesimal generator is $X=-\alpha(\partial / \partial \alpha)+x \ln x(\partial / \partial x), \bar{\alpha}=$ $\exp \{-g[\alpha \partial / \partial \alpha-x \ln x(\partial / \partial x)] \alpha\}=\exp [-g \alpha \partial / \partial \alpha] \alpha=\alpha-g \alpha+g^{2} \alpha / 2 !$ $-g^{3} \alpha / 3 ! \cdots=\alpha e^{-g}, \bar{x}=\exp \left[g x \ln x(\partial / \partial x] x=\sum_{m}(1 / m !)[g x \ln x(\partial / \partial x)]^{m} x\right.$. 
Let $\ln \ln x=y, \bar{x}=\sum_{m}(1 / m !)(g \partial / \partial y)^{m} \exp \left(e^{y}\right)=\exp \left(e^{y+g}\right)=x^{e \theta}$, i.e., the local group is given by $\bar{\alpha}=c \alpha, \bar{x}=x^{1 / c}, c>0$, which is actually a group.

EXAMPLE 2. A problem that is of interest in the field of statistical inference is to find out if the c.d.f. of the correlation coefficient $r$, estimated from a sample of size $N$ from a bivariate normal distribution with correlation coefficient $\rho$ is invariant under some group of transformations. This problem is of interest for two reasons, first if such a group exists and it is locally compact the Haar measure on it provides a particularly appealing prior measure to use in the application of Bayes' theorem. Secondly if the group exists and it satisfies certain properties a fiducial distribution for $\rho$ may be constructed following Fraser [4] [5] and it would be of interest to compare this fiducial distribution with the one given by Fisher in his original paper on fiducial probability [3].

The density of the correlation coefficient may be written as,

$$
f_{n}(r, \rho)=\frac{2^{n-2}\left(1-\rho^{2}\right)^{\frac{1}{2} n}\left(1-r^{2}\right)^{\frac{1}{2}(n-3)}}{(n-2) ! \pi} \sum_{\alpha=0}^{\infty} \frac{(2 \rho r)^{\alpha}}{\alpha !} \Gamma^{2}\left[\frac{1}{2}(n+\alpha)\right]
$$

where $n=N-1$. (See [1].)

The c.d.f. will be invariant under a Lie transformation group only if there exist differentiable functions $a(r), b(\rho)$ such that,

$$
\frac{d}{d r}\left[a(r) f_{n}(r, \rho)\right]=b(\rho) \frac{d}{d \rho} f_{n}(r, \rho) .
$$

Write $f_{n}$ as, $K_{n}\left(1-\rho^{2}\right)^{\frac{1}{2} n}\left(1-r^{2}\right)^{\frac{1}{2}(n-3)} C(2 \rho r)$. Therefore

$$
\begin{aligned}
& \left(1-\rho^{2}\right)^{\frac{1}{2} n} \frac{d}{d r}\left[a(r)\left(1-r^{2}\right)^{\frac{1}{2}(n-3)} C(2 \rho r)\right] \\
& \quad=b(\rho)\left(1-r^{2}\right)^{\frac{1}{2}(n-3)}\left[\left(1-\rho^{2}\right)^{\frac{1}{3} n} C^{\prime}(2 \rho r) 2 r-\left(1-\rho^{2}\right)^{\frac{1}{2} n-1} n \rho C(2 \rho r)\right] .
\end{aligned}
$$

Set $\rho=0$

$$
C(0) \frac{d}{d r}\left[a(r)\left(1-r^{2}\right)^{\frac{1}{2}(n-3)}\right]=b(0)\left(1-r^{2}\right)^{\frac{1}{2}(n-3)} C^{\prime}(0) 2 r .
$$

Therefore,

$$
C(0) a(r)\left(1-r^{2}\right)^{\frac{1}{2}(n-3)}=-b(0)\left(1-r^{2}\right)^{\frac{1}{2(n-1)}} C^{\prime}(0) 2 /(n-1)+K .
$$

Equation (5) becomes

(6) $\left(1-\rho^{2}\right)\left[b(0) C^{\prime}(0) 2 r C(2 \rho r) / C(0)\right.$

$$
\begin{aligned}
\left.-b(0)\left(1-r^{2}\right) C^{\prime}(0) 4 \rho C^{\prime}(2 \rho r) / C(0)(n-1)+2 \rho K C^{\prime}(2 \rho r) / C(0)\right] \\
=b(\rho)\left[\left(1-\rho^{2}\right) C^{\prime}(2 \rho r) 2 r-n \rho C(2 \rho r)\right] .
\end{aligned}
$$

Set $r=0$, yielding $b(\rho)=\left(1-\rho^{2}\right) \times$ a constant.

Setting $\rho=0$ the constant $=b(0)$, i.e., $b(\rho)=\left(1-\rho^{2}\right) b(0)$. Substitute in (6), 


$$
\begin{aligned}
& b(0) C^{\prime}(0) 2 r C^{\prime}(2 \rho r) / C(0) \\
& -b(0)\left(1-r^{2}\right) C^{\prime}(0) 4 \rho C^{\prime}(2 \rho r) / C(0)(n-1)+2 \rho K C^{\prime}(2 \rho r) \\
& =\left(1-\rho^{2}\right) b(0)\left[\left(1-\rho^{2}\right) C^{\prime}(2 \rho r) 2 r-n \rho C(2 \rho r)\right] .
\end{aligned}
$$

Equating the coefficient of $\rho r^{2}$ on both sides of (7),

$$
\begin{aligned}
b(0) C^{\prime}(0) \Gamma^{2}\left[\frac{1}{2}(n+1)\right] / C(0)+b(0) C^{\prime}(0) \Gamma^{2}\left[\frac{1}{2}(n+1)\right] / C(0)(n-1) \\
=b(0) \Gamma^{2}\left[\frac{1}{2}(n+2)\right] .
\end{aligned}
$$

Therefore $b(0)=0$ or,

$$
\Gamma^{4}\left[\frac{1}{2}(n+1)\right] n /(n-1)=\Gamma^{2}\left[\frac{1}{2}(n+2)\right] \Gamma^{2}\left[\frac{1}{2} n\right] .
$$

The latter is easily seen to be impossible, consequently $b(0)=0$ implying $b(\rho)=0$. It therefore follows that the c.d.f. of the correlation coefficient is invariant under no Lie transformation group.

Lindley in [10] proved a theorem to the effect that for real random variables a fiducial distribution is a Bayes' posterior distribution if and only if the problem is invariant.

Applying this theorem here one can now say that the fiducial distribution for $\rho$ is not a Bayes' posterior distribution for any prior distribution.

This example also demonstrates that a possibility suggested to me by Dr. J. Berkson is not true in general. Namely, that when the fiducial distribution provides a frequency interpretable probability, that probability is the one given by the Bayes' formula with a uniform distribution of the prior probabilities.

EXAMPLE 3. In [2] an example is given to demonstrate that the above mentioned theorem of Lindley does not extend to spaces of dimension higher than one. Unfortunately the theorem quoted in [2], from which it follows that the given example is in fact a counterexample, is not general enough. The theorem proved above is of sufficient generality. Doubtlessly the given example still provides a counterexample; however it seems useful to give an example to which the above theorem may be applied more easily.

Consider

$$
f(x, y, \alpha, \beta)=(2 \pi)^{-1} \sigma(\alpha) \exp \left[-\frac{1}{2}(x-\alpha)^{2}\right] \exp \left[-\frac{1}{2} \sigma^{2}(\alpha)(y-\beta)^{2}\right]
$$

where $\sigma(\alpha)$ is selected as in [2]. One may easily verify that Condition (1) of the theorem above leads to a contradiction.

I would like to thank David Lowdenslager for looking over a section of this paper, and the referee for a number of suggestions.

\section{REFERENCES}

[1] Anderson, T. W. (1958). Introduction to Multivariate Statistical Analysis. Wiley, New York.

[2] Brillinger, D. R. (1962). Examples bearing on the definition of fiducial probability with a bibliography. Ann. Math. Statist. 33 1349-1355.

[3] Fisher, R. A. (1930). Inverse probability. Proc. Cambridge Philos. Soc. 26 528-535. 
[4] Fraser, D. A. S. (1961). The fiducial method and invariance. Biometrika 48 261-280.

[5] Fraser, D. A. S. (1961). On fiducial inference. Ann. Math. Statist. 32 661-676.

[6] Kiffer, J. (1957). Invariance, minimax sequential estimation, and continuous time processes. Ann. Math. Statist. 28 573-601.

[7] Kudo, H. (1955). On minimax invariant estimates of the transformations parameter. Nat. Sci. Rep. Ochanomizu Univ. 6 31-73.

[8] Lehmann, E. L. and Stein, C. (1953). The totality of transformations leaving a family of normal distributions invariant (abstract). Ann. Math. Statist. 24142.

[9] Lehmann, E. L. (1959). Testing Statistical Hypotheses. Wiley, New York.

[10] Lindley, D. V. (1959). Fiducial distributions and Bayes' theorem. J. Roy. Stat. Soc. Ser. $B 21$ 102-107.

[11] Palais, R. S. (1957). Global Formulation of the Lie Theory of Transformation Groups. Amer. Math. Soc. Memoir.

[12] Peisakoff, M. (1951). Transformation parameters. Unpublished Princeton thesis.

[13] Pitman, E. J. G. (1939). Tests of hypotheses concerning location and scale parameters. Biometrika 31 200-215.

[14] Pitman, E. J. G. (1939). Location and scale parameters. Biometrika 30 391-421.

[15] WeNDEL, J. G. (1957). Invariance of normal distributions. Michigan Math. J. 4 173-174.

[16] Wesler, O. (1959). Invariance theory and a modified minimax principle. Ann. Math. Statist. 30 1-20. 